\title{
Nasal Carriage of Staphylococci among Health Care Workers and Impact of Conventional Decolonisation Methods
}

https://doi.org/10.47210/bjohns.2021.v29i1.426

Chiranjib Das, ${ }^{1}$ Ranadeep Ghosh, ${ }^{2}$ Kingshuk Dhar ${ }^{2}$

$\underline{\text { Introduction }}$

\section{ABSTRACT}

Staphylococci are one of the most common causes of nosocomial infections. The principal route of transmission of Staphylococci is the contaminated hands of health care workers (HCWs). Staphylococci can also be found as part of the nasal microbiota without causing overt disease. So we undertake the present study to estimate the prevalence of asymptomatic nasal carriage of Staphylococci among HCWs and impact of conventional decolonisation methods in a tertiary care hospital in West Bengal. Materials and Methods

Nasal swabs were collected from anterior nares of HCWs for culture and antibiotic sensitivity test on day one. HCWs who were found to be carriers of Staphylococci were advised to apply mupirocin ointment to anterior nares twice daily along with chlorhexidine gluconate bath once daily for five days. All HCWs were also advised to practice standard hygiene protocol. All of them were re-tested for nasal swab culture and antibiotic sensitivity on day seven and day twenty eight.

$\underline{\text { Results }}$

Nasal carriage of Staphylococci in the first, second and third culture report was found to be $64.28 \%, 7.14 \%$ and $24.49 \%$ respectively. Cefotaxime, cotrimoxazole and erythromycin were least effective against Staphylococci. There was variable sensitivity to clindamycin, gentamycin and ciprofloxacin. All strains of Staphylococci were highly sensitive to linezolid. All strains of Staphylococci except MRSA were highly sensitive to vancomycin.

\section{Conclusion}

The present study re-establishes the fact that HCWs carry Staphylococci in their nose in significantly high proportion. So different measures should be undertaken to minimise Staphylococci related nosocomial infections.

$\underline{\text { Kevwords }}$

Staphylococci; Nosocomial Infection; Health Care Workers; Nasal Carriage; Decolonisation

$\mathrm{N}$ osocomial infections represent an important health problem in terms of morbidity, mortality and cost of treatment. Prevention of these infections is a key priority. Staphylococci are one of the most common causes of nosocomial infections. ${ }^{1}$ The

1 - Department of ENT, Maharaja Jitendra Narayan Medical College and Hospital, Cooch Behar, West Bengal 2 - Department of Microbiology, Maharaja Jitendra Narayan Medical College and Hospital, Cooch Behar, West Bengal

\section{Corresponding author:}

Dr Chiranjib Das

email: chirubata.das.87@gmail.com principal route of transmission of Staphylococci in the hospital is from patient to patient via the contaminated hands of health care workers (HCWs). ${ }^{2}$ Staphylococci can also be found as part of the nasal microbiota without causing overt disease.

This carrier state is an important factor for dissemination from $\mathrm{HCW}$ s to patients and vice-versa. ${ }^{3,4}$ Another challenge associated with Staphylococci is the development of multidrug resistant strains to various available antibiotics. ${ }^{5}$ So we undertake the present study to estimate the prevalence of asymptomatic nasal carriage of Staphylococci among HCWs and impact of conventional decolonisation methods in a tertiary care hospital in West Bengal. 


\section{Materials and Methods}

We conducted a prospective study among HCWs over a period of one month from 1st July 2019 to 31st July 2019 in a tertiary care hospital in the northern part of West Bengal. Institutional Ethical Committee clearance and informed consent from each participant were taken. HCWs from medicine ward, surgery ward and critical care unit (CCU) were recruited in the study. HCWs comprised of doctors, nurses and group-D staff. HCWs suffering from fever, upper respiratory tract infection, impetigo, skin and subcutaneous infections, diabetes mellitus, immunocompromisation, were excluded from the present study. HCWs with history of recent nasal surgery, use of nasal antiseptics, or antimicrobial therapy, an MRSA decolonization attempt in the previous 6 months, allergy to mupirocin or chlorhexidine, were also excluded from the study. HCWs who were pregnant, breast feeding, or did not turn up in the follow up were also excluded.

Nasal swabs were collected from anterior nares of the participants using sterile cotton swabs moistened with sterile normal saline on day one. The swabs were then immediately transported with aseptic precautions to the Microbiology laboratory. Specimens were inoculated on $10 \%$ sheep blood agar, Nutrient agar and MacConkey's agar plates and incubated overnight at $37^{\circ} \mathrm{C}$. Samples were identified by standard methods based on colony morphology, pigment production, Gram staining, catalase test, slide coagulase test, modified Hugh and Leifson $(\mathrm{O} / \mathrm{F})$ test and fermentation of mannitol. All of the isolated Staphylococci strains were tested against different antimicrobial agents by the modified Kirby Bauer disc diffusion method on Mueller Hinton agar following Clinical and Laboratory Standards Institute guidelines. The antibiotic discs used were clindamycin (CLIND) $2 \mathrm{mcg}$, gentamicin (GEN) $10 \mathrm{mcg}$, cefotaxime (CEFO) $30 \mathrm{mcg}$, ciprofloxacin (CIPRO) $10 \mathrm{mcg}$, cotrimoxazole (COTRI) $25 \mathrm{mcg}$, erythromycin (ERY) $15 \mathrm{mcg}$, linezolid (LIN) $30 \mathrm{mcg}$, and vancomycin (VAN) 30mcg. Methicillin resistant Staphylococci were detected by using cefoxitin $30 \mathrm{mcg}$ discs.

$\mathrm{HCW}$ s who were found to be carriers of Staphylococci in the first culture were advised to apply mupirocin ointment to anterior nares twice daily along with chlorhexidine gluconate bath once daily for five days. All HCWs were also advised to practice standard hygiene protocol including hand washing before and after patient examination, use of sterile aprons, gloves and masks, and avoiding touching one's nose during work. All of them were re-tested for nasal swab culture and antibiotic sensitivity on day seven and day twenty eight.

\section{Results}

Total 98 participants were included in the present study. Distribution of methicillin sensitive Staphylococcus aureus (MSSA), methicillin resistant Staphylococcus aureus (MRSA), methicillin sensitive coagulase negative Staphylococci (MS CoNS), and methicillin resistant CoNS (MR CoNS) in the nasal swab culture on day one, seven and twenty eight are shown in Table I. In the first, second and third culture report nasal carriage of Staphylococci was found to be $64.28 \%, 7.14 \%$ and $24.49 \%$ respectively.

Comparative distribution of nasal carriage of Staphylococci among the different HCWs in different wards on day one, seven, and twenty eight are depicted in Tables II, III and IV respectively. There were 27 doctors, 58 nurses and 13 group-D staffs in the present study. Nasal carriage of Staphylococci in doctors on day one, seven and twenty eight was $66.67 \%, 3.70 \%$ and $22.22 \%$ respectively. Nasal carriage of Staphylococci in nurses on day one, seven and twenty eight was $67.24 \%, 8.62 \%$ and $27.59 \%$ respectively. Nasal carriage of Staphylococci in group-D staff on day one, seven and twenty eight was $46.15 \%, 7.69 \%$ and $15.38 \%$ respectively. Nasal carriage of Staphylococci among HCWs in medicine ward on day one, seven and twenty eight was $51.22 \%, 2.44 \%$ and $12.20 \%$ respectively. Nasal carriage of Staphylococci among HCWs in surgery ward on day one, seven and twenty eight was $72.5 \%, 7.5 \%$ and $35 \%$ respectively. Nasal carriage of Staphylococci among HCWs in CCU on day one, seven and twenty eight was $76.47 \%, 17.65 \%$ and $29.41 \%$ respectively.

Antibiotic sensitivity of MSSA, MRSA, MS CoNS, and MR CoNS on day one, seven and twenty eight are shown in Tables V, VI and VII respectively. 
Table I: Distribution of samples according to culture report on day one, seven and twenty eight

\begin{tabular}{|c|c|c|c|}
\hline \multirow{2}{*}{ ISOLATE } & \multicolumn{3}{|c|}{ NUMBER (PERCENTAGE) } \\
\hline MSSA & DAY 1 & DAY 7 & DAY 28 \\
\hline MRSA & $19(19.38)$ & $\mathbf{0}(0)$ & $\mathbf{2}(2.04)$ \\
\hline MS CoNS & $5(5.1)$ & $1(1.02)$ & $5(5.1)$ \\
\hline MR CoNS & $37(37.76)$ & $\mathbf{5}(5.1)$ & $\mathbf{1 5}(15.31)$ \\
\hline Others & $2(2.04)$ & $\mathbf{2}(\mathbf{2 . 0 4})$ & $4(4.08)$ \\
\hline No growth & $14(14.29)$ & $\mathbf{8 9}(90.82)$ & $70(71.43)$ \\
\hline
\end{tabular}

MSSA:methicillin sensitive Staphylococcus aureus, MRSA:methicillin resistant Staphylococcus aureus, MS CoNS:methicillin sensitive coagulase negative Staphylococci, MR CoNS:methicillin resistant coagulase negative Staphylococci

Table II: Distribution of nasal carriage of Staphylococci among the different HCWs in different wards on day one

\begin{tabular}{|c|c|c|c|c|c|c|c|c|}
\hline \multirow{4}{*}{ ISOLATE } & \multicolumn{8}{|c|}{ NUMBER (PERCENTAGE) } \\
\hline & \multirow{2}{*}{\multicolumn{2}{|c|}{$\begin{array}{c}\text { DOCTORS } \\
(\mathbf{N}=\mathbf{2 7})\end{array}$}} & \multirow{2}{*}{\multicolumn{3}{|c|}{$\begin{array}{l}\text { NURSES } \\
(\mathbf{N}=\mathbf{5 8})\end{array}$}} & \multirow{2}{*}{\multicolumn{3}{|c|}{$\begin{array}{l}\text { GROUP-D STAFF } \\
\qquad(\mathrm{N}=13)\end{array}$}} \\
\hline & & & & & & & & \\
\hline & $\begin{array}{l}\text { MEDICINE } \\
\text { WARD } \\
\text { (N=14) }\end{array}$ & $\begin{array}{l}\text { SURGERY } \\
\text { WARD } \\
(\mathbf{N}=13)\end{array}$ & $\begin{array}{l}\text { MEDICINE } \\
\text { WARD } \\
(\mathbf{N}=\mathbf{2 2})\end{array}$ & $\begin{array}{l}\text { SURGERY } \\
\text { WARD } \\
\text { (N=22) }\end{array}$ & $\begin{array}{c}\text { CCU } \\
(\mathrm{N}=14)\end{array}$ & $\begin{array}{l}\text { MEDICINE } \\
\text { WARD } \\
(\mathbf{N}=\mathbf{5})\end{array}$ & $\begin{array}{l}\text { SURGERY } \\
\text { WARD } \\
(\mathbf{N}=\mathbf{5})\end{array}$ & $\begin{array}{c}\mathrm{CCU} \\
(\mathrm{N}=\mathbf{3})\end{array}$ \\
\hline MSSA & 3 & 3 & 3 & 4 & 3 & 1 & 2 & $\mathbf{0}$ \\
\hline MRSA & 1 & 1 & $\mathbf{0}$ & 1 & 1 & $\mathbf{0}$ & $\mathbf{0}$ & 1 \\
\hline MS CoNS & 4 & 6 & 9 & 11 & 5 & $\mathbf{0}$ & 1 & 1 \\
\hline MR CoNS & $\mathbf{0}$ & $\mathbf{0}$ & $\mathbf{0}$ & $\mathbf{0}$ & 2 & $\mathbf{0}$ & $\mathbf{0}$ & $\mathbf{0}$ \\
\hline
\end{tabular}

MSSA:methicillin sensitive Staphylococcus aureus, MRSA:methicillin resistant Staphylococcus aureus, MS CoNS:methicillin sensitive coagulase negative Staphylococci, MR CoNS:methicillin resistant coagulase negative Staphylococci 
Table III: Distribution of nasal carriage of Staphylococci among the different HCWs in different wards on day seven

\begin{tabular}{|c|c|c|c|c|c|c|c|c|}
\hline \multirow{4}{*}{ ISOLATE } & \multicolumn{8}{|c|}{ NUMBER (PERCENTAGE) } \\
\hline & \multirow{2}{*}{\multicolumn{2}{|c|}{$\begin{array}{c}\text { DOCTORS } \\
(\mathbf{N}=27)\end{array}$}} & \multirow{2}{*}{\multicolumn{3}{|c|}{$\begin{array}{c}\text { NURSES } \\
(\mathbf{N}=\mathbf{5 8})\end{array}$}} & \multirow{2}{*}{\multicolumn{3}{|c|}{$\begin{array}{l}\text { GROUP-D STAFF } \\
\qquad(\mathrm{N}=13)\end{array}$}} \\
\hline & & & & & & & & \\
\hline & $\begin{array}{l}\text { MEDICINE } \\
\text { WARD } \\
(\mathrm{N}=14)\end{array}$ & $\begin{array}{l}\text { SURGERY } \\
\text { WARD } \\
(\mathbf{N}=13)\end{array}$ & $\begin{array}{l}\text { MEDICINE } \\
\text { WARD } \\
(\mathbf{N}=\mathbf{2 2})\end{array}$ & $\begin{array}{c}\text { SURGERY } \\
\text { WARD } \\
(\mathbf{N}=\mathbf{2 2})\end{array}$ & $\underset{(\mathrm{N}=14)}{\mathrm{CCU}}$ & $\begin{array}{c}\text { MEDICINE } \\
\text { WARD } \\
(\mathrm{N}=5)\end{array}$ & $\begin{array}{c}\text { SURGERY } \\
\text { WARD } \\
(\mathrm{N}=5)\end{array}$ & $\begin{array}{l}\mathbf{C C U} \\
(\mathbf{N}=\mathbf{3})\end{array}$ \\
\hline MSSA & 0 & $\mathbf{0}$ & 0 & $\mathbf{0}$ & $\mathbf{0}$ & $\mathbf{0}$ & $\mathbf{0}$ & $\mathbf{0}$ \\
\hline MRSA & $\mathbf{0}$ & $\mathbf{0}$ & $\mathbf{0}$ & 1 & $\mathbf{0}$ & $\mathbf{0}$ & $\mathbf{0}$ & $\mathbf{0}$ \\
\hline MS CoNS & $\mathbf{0}$ & 1 & 1 & 1 & 1 & $\mathbf{0}$ & $\mathbf{0}$ & 1 \\
\hline MR CoNS & $\mathbf{0}$ & $\mathbf{0}$ & $\mathbf{0}$ & $\mathbf{0}$ & 1 & $\mathbf{0}$ & $\mathbf{0}$ & $\mathbf{0}$ \\
\hline
\end{tabular}

MSSA:methicillin sensitive Staphylococcus aureus, MRSA:methicillin resistant Staphylococcus aureus, MS CoNS:methicillin sensitive coagulase negative Staphylococci, MR CoNS:methicillin resistant coagulase negative Staphylococci

Table IV: Distribution of nasal carriage of Staphylococci among the different HCWs in different wards on day twenty eight

\begin{tabular}{|c|c|c|c|c|c|c|c|c|}
\hline \multirow{4}{*}{ ISOLATE } & \multicolumn{8}{|c|}{ NUMBER (PERCENTAGE) } \\
\hline & \multirow{2}{*}{\multicolumn{2}{|c|}{$\begin{array}{l}\text { DOCTORS } \\
(\mathbf{N}=\mathbf{2 7})\end{array}$}} & \multirow{2}{*}{\multicolumn{3}{|c|}{$\begin{array}{c}\text { NURSES } \\
(\mathbf{N}=\mathbf{5 8})\end{array}$}} & \multirow{2}{*}{\multicolumn{3}{|c|}{$\begin{array}{l}\text { GROUP-D STAFF } \\
\qquad(\mathrm{N}=13)\end{array}$}} \\
\hline & & & & & & & & \\
\hline & $\begin{array}{l}\text { MEDICINE } \\
\text { WARD } \\
(\mathbf{N}=\mathbf{1 4})\end{array}$ & $\begin{array}{l}\text { SURGERY } \\
\text { WARD } \\
(\mathbf{N}=13)\end{array}$ & $\begin{array}{l}\text { MEDICINE } \\
\text { WARD } \\
(\mathbf{N}=\mathbf{2 2})\end{array}$ & $\begin{array}{l}\text { SURGERY } \\
\text { WARD } \\
(\mathbf{N}=\mathbf{2 2})\end{array}$ & $\begin{array}{c}\mathrm{CCU} \\
(\mathrm{N}=14)\end{array}$ & $\begin{array}{l}\text { MEDICINE } \\
\text { WARD } \\
(\mathbf{N}=\mathbf{5})\end{array}$ & $\begin{array}{c}\text { SURGERY } \\
\text { WARD } \\
(\mathbf{N}=\mathbf{5})\end{array}$ & $\begin{array}{l}\mathrm{CCU} \\
(\mathrm{N}=\mathbf{3})\end{array}$ \\
\hline MSSA & $\mathbf{0}$ & 1 & $\mathbf{0}$ & $\mathbf{0}$ & 1 & $\mathbf{0}$ & $\mathbf{0}$ & $\mathbf{0}$ \\
\hline MRSA & 0 & 1 & 1 & 2 & 0 & 0 & 1 & 0 \\
\hline MS CoNS & 1 & 3 & 3 & 5 & 2 & $\mathbf{0}$ & $\mathbf{0}$ & 1 \\
\hline MR CoNS & 0 & $\mathbf{0}$ & $\mathbf{0}$ & 1 & 1 & $\mathbf{0}$ & $\mathbf{0}$ & $\mathbf{0}$ \\
\hline
\end{tabular}

MSSA:methicillin sensitive Staphylococcus aureus, MRSA:methicillin resistant Staphylococcus aureus, MS CoNS:methicillin sensitive coagulase negative Staphylococci, MR CoNS:methicillin resistant coagulase negative Staphylococci 
Clindamycin and gentamycin were only effective against MS CoNS. Ciprofloxacin was effective against all strains of Staphylococci except MRSA. All strains of Staphylococci were highly sensitive to linezolid. All strains of Staphylococci except MRSA were highly sensitive to vancomycin. Cefotaxime, cotrimoxazole and erythromycin were least effective against Staphylococci.

There was no adverse effect of the topical decolonisation agents among the participants.

\section{Discussion}

Worldwide, most of the literature focuses on carriage of Staphylococcus aureus and impact of decolonisation methods among patients. Other Staphylococci such as (CoNS) are also pathogenic. Methicillin resistant CoNS (MRCoNS) have also been found worldwide. Moreover, CoNS may transfer its resistance to $\mathrm{MRSA}^{6,7}$. HCWs are at the interface between hospitals and communities ${ }^{8}$. So periodic screening of HCWs to identify carrier state and measures taken to decolonise them is crucial in prevention of Staphylococci associated nosocomial infection.
Nasal carriage of Staphylococci among HCWs was $64.28 \%$ in the present study. It comprised of $24.48 \%$ Staphylococcus aureus and 39.8\% CoNS. In a similar study by Nadia. E. Al-Abdli et al, nasal carriage of Staphylococci was $83.9 \%$, comprising of $47.5 \%$ Staphylococcus aureus and $36.4 \%$ CoNS. ${ }^{9}$ Staphylococcus aureus and MRSA carriage rate among HCWs in the present study are similar to the internationally reported range which are $19.80 \%$ to $48 \%$ and $5.8 \%$ to $17.8 \%$ respectively. ${ }^{10,11}$ This wide range can be attributed to variations in sampling technique, culture and method of MRSA identification, local infection control standards and the local prevalence of MRSA.

In the present study nasal carriage of Staphylococci was highest among nurses (67.24\%) and doctors (66.67\%) followed by group-D staffs $(46.15 \%)$. Nasal carriage of Staphylococci was highest among HCWs of CCU (76.47\%) and surgery ward (72.5\%) followed by medicine ward $(51.22 \%)$. Similar findings were noted in other studies also. ${ }^{12}$ Higher rate of nasal carriage of Staphylococci in specific groups of HCWs can be due to frequent contact with infected wounds in specific wards.

Table V: Antibiotic sensitivity of Staphylococci on day one

\begin{tabular}{|c|c|c|c|c|c|c|c|c|}
\hline \multirow{2}{*}{ ORGANISM } & \multicolumn{7}{c|}{ ANTIBIOTIC SENSITIVITY (IN PERCENTAGE) } \\
\cline { 2 - 8 } & CLIND & GEN & CEFO & CIPRO & COTRI & ERY & LIN & VAN \\
\hline MSSA & 57.89 & 52.63 & 52.63 & 73.68 & 36.84 & 46.37 & 100 & 94.74 \\
\hline MRSA & 40 & 20 & 0 & 40 & 20 & 0 & 100 & 60 \\
\hline MS CoNS & 83.78 & 75.68 & 59.46 & 67.57 & 54.05 & 48.65 & 100 & 100 \\
\hline MR CoNS & 50 & 50 & 0 & 100 & 50 & 50 & 100 & 100 \\
\hline
\end{tabular}

CEFO:cefotaxime, COTRI:cotrimoxazole, ERY:erythromycin CLIND:Clindamycin, GEN:gentamycin, CIPRO:ciprofloxacin, LIN:linezolid, VAN:vancomycin, MSSA:methicillin sensitive Staphylococcus aureus, MRSA:methicillin resistant Staphylococcus aureus, MS CoNS:methicillin sensitive coagulase negative Staphylococci, MR CoNS:methicillin resistant coagulase negative Staphylococci 
Table VI: Antibiotic sensitivity of Staphylococci on day seven

\begin{tabular}{|c|c|c|c|c|c|c|c|c|}
\hline \multirow{2}{*}{ ORGANISM } & \multicolumn{8}{|c|}{ ANTIBIOTIC SENSITIVITY (IN PERCENTAGE) } \\
\hline & CLIND & GEN & CEFO & CIPRO & & ERY & LIN & VAN \\
\hline MRSA & $\mathbf{0}$ & $\mathbf{0}$ & $\mathbf{0}$ & $\mathbf{0}$ & $\mathbf{0}$ & $\mathbf{0}$ & 100 & $\mathbf{0}$ \\
\hline MS CoNS & 80 & 48 & 60 & 80 & 60 & 60 & 100 & 100 \\
\hline MR CoNS & $\mathbf{0}$ & $\mathbf{0}$ & $\mathbf{0}$ & 100 & $\mathbf{0}$ & $\mathbf{0}$ & 100 & 100 \\
\hline
\end{tabular}

Table VII: Antibiotic sensitivity of Staphylococci on day twenty eight

\begin{tabular}{|c|c|c|c|c|c|c|c|c|}
\hline \multirow{2}{*}{ ORGANISM } & \multicolumn{7}{c|}{ ANTIBIOTIC SENSITIVITY (IN PERCENTAGE) } \\
\cline { 2 - 7 } & CLIND & GEN & CEFO & CIPRO & & ERY & LIN \\
\hline MSSA & 50 & 50 & 0 & 50 & 0 & 0 & 100 \\
\hline MRSA & 40 & 60 & 0 & 60 & 20 & 20 & 100 & 60 \\
\hline MS CoNS & 66.67 & 73.33 & 53.33 & 73.33 & 40 & 46.67 & 100 & 100 \\
\hline MR CoNS & 100 & 50 & 0 & 100 & 0 & 0 & 100 & 100 \\
\hline
\end{tabular}

CEFO:cefotaxime, COTRI:cotrimoxazole, ERY:erythromycin CLIND:Clindamycin, GEN:gentamycin, CIPRO:ciprofloxacin, LIN:linezolid, VAN:vancomycin, MSSA:methicillin sensitive Staphylococcus aureus, MRSA:methicillin resistant Staphylococcus aureus, MS CoNS:methicillin sensitive coagulase negative Staphylococci, MR CoNS:methicillin resistant coagulase negative Staphylococci

United States Food and Drug Administration approved mupirocin for decolonization of the anterior nares. ${ }^{13}$ It is a topical anti-staphylococcal antibiotic. Nasal carriers of Staphylococci may also harbour the organism at various extra-nasal sites. ${ }^{14}$ It is unlikely that nasal application of mupirocin will have any effect on these sites. Decolonization of the skin can be achieved by washing with chlorhexidine gluconate. ${ }^{15}$ The combination of nasal mupirocin ointment along with chlorhexidine bath was preferred over other alternative agents due to strong evidences generated in favour of this combination for reduction of MRSA burden and decolonization of MRSA carriers in Cochrane review, ${ }^{16}$ and meta- analysis ${ }^{17}$ in recent times. Moreover, education about both hygiene and regular environmental disinfection measures has also been included to reduce carriage and prevent infection. Simple preventive measures like hand washing before and after patient examination, use of sterile aprons, gloves and masks, awareness during the examination of immunocompromised patients, and avoiding touching one's nose during work, can reduce transmission of Staphylococci considerably.

In the present study, HCWs found to carry nasal Staphylococci on first culture report were advised to apply mupirocin ointment to anterior nares twice daily and chlorhexidine gluconate bath once daily for five days 
along with maintenance of standard hygiene protocol. After seven days nasal carriage of Staphylococci were found to decrease from $64.28 \%$ to $7.14 \%$. After twenty eight days, it was found to increase to $24.49 \%$. This increase may be due to various factors. The HCWs might be re-exposed and become colonized with the same or a new strain of Staphylococci There is also possibility that some HCWs did not follow the hygiene protocol strictly.

Another major issue is the emergence of multidrug resistant Staphylococci. ${ }^{18}$ This is due to misuse of cheap and easily available over-the-counter antibiotics. This causes infections which are difficult to treat which in turn prolongs hospitalization and cost of treatment. In the present study cefotaxime, cotrimoxazole and erythromycin were least effective against Staphylococcus. Clindamycin, gentamycin and ciprofloxacin were effective against limited number of samples. Even vancomycin was not effective against all MRSA. Only linezolid was effective against all samples.

\section{Conclusion}

The present study re-establishes the fact that HCWs carry Staphylococci in their nose in significantly high proportion. So periodic screening of HCWs for their carrier state should be done for their own sake, as well as for patients and community as a whole. Decolonisation measures should be taken for carriers of Staphylococci. But this doesn't eliminate the chance of re-infection. HCWs should be periodically educated and trained about the maintenance of personal hygiene measures to be followed within hospital premises. Community awareness programmes on the effects of use or rather the misuse of antibiotics should be held from time to time. Apart from this, time to time disinfection of the healthcare setting may be carried out as per the institutional protocol, to prevent re-colonization by Staphylococci among HCWs.

\section{References}

1. Wisplinghoff H, Bischoff T, Tallent SM, Seifert H, Wenzel RP, Edmond MB. Nosocomial bloodstream infections in US hospitals: analysis of 24,179 cases from a prospective nationwide surveillance study. Clin Infect Dis. 2004; 39:309-17

2. Solberg CO. Spread of Staphylococcus aureus in hospitals: causes and prevention. Scand J Infect Dis. 2000; 32:587-95

3. Cookson B, Peters B, Webster M, Phillips I, Rahman M, Noble W. Staff carriage of epidemic methicillin-resistant Staphylococcus aureus. J Clin Microbiol. 1989; 27:1471-6

4. David MZ, Daum RS. Community-associated methicillinresistant Staphylococcus aureus: epidemiology and clinical consequences of an emerging epidemic. Clin Microbiol Rev. 2010; 23:616-87

5. S. Bruzzese , K. Bush, J. Leal, J. Kim , D.M. Vickers , A. Rusk, et al. Comparing the epidemiology of hospital-acquired methicillin-resistant Staphylococcus aureus clone groups in Alberta, Canada. Epidemiol. Infect. 2016;144(10);2184-90

6. Sefani, S and Varaldo, P.E. Epidemiology of methicillin resistant staphylococci in Europe. Clin. Microbiol. Infect. 2003,9:1179-86

7. Hurdle, J.G, O'Neill, A.J, Mody, L, Chopra, I and Bradely, S.F. In vivo transfer of high level mupirocin resistant Staphylococcus aureus associated with failure of mupirocin prophylaxisis. J. Antimicrob. Chemother. 2005,56:1166-8

8. Albrich WC, Harbarth S. Health-care workers: source, vector, or victim of MRSA? Lancet Infect Dis. 2008;8:289-301

9. Nadia. E. Al-Abdli, and Saleh.H. Baiu, "Nasal Carriage of Staphylococcus in Health Care Workers in Benghazi Hospitals." American Journal of Microbiological Research 2014; 2(4): $110-2$

10. Na'was T, Fakhoury J. Nasal carriage of methicillin-resistant Staphylococcus aureus by hospital staff in north Jordan. J Hosp Infect. 1991; 17:223-9

11. AkouaKoffi C, Dje K, Toure R. Nasal carriage of methicillin resistant Staphylococcus aureus among health care personnel in Abidjan (Cote d'Ivoire). Dakar Med. 2004;49:70-4

12. Shibabaw A, Abebe T, Mihret A. Nasal carriage rate of methicillin resistant Staphylococcus aureus among Dessie Referral Hospital Health Care Workers; Dessie, Northeast Ethiopia. Antimicrobial Resist Infect Control. 2013; 2:25

13. Perl TM, Cullen JJ, Wenzel RP, Zimmerman MB, Pfaller MA, Sheppard D, Twombley J, French PP, Herwaldt LA. Mupirocin and the Risk of Staphylococcus aureus Study Team. Intranasal mupirocin to prevent postoperative Staphylococcus aureus infections. N Engl J Med. 2002;346:1871-7

14. Wertheim HF, Melles DC, Vos MC, et al. The role of nasal carriage in Staphylococcus aureus infections. Lancet Infect Dis. 2005;5:751-62

15. Kaiser AB, Kernodle DS, Barg NL, Petracek MR. Influence of 
preoperative showers on staphylococcal skin colonization: a comparative trial of antiseptic skin cleansers. Ann Thorac Surg. 1988;45:35-8

16. Van Rijen M, Bonten M, Wenzel R, Kluytmans J. Mupirocin ointment for preventing Staphylococcus aureus infections in nasal carriers. Cochrane Database Syst Rev. 2008 Oct
8;(4):CD006216. doi: 10.1002/14651858.CD006216.pub2

17. Septimus EJ, Schweizer ML. Decolonization in Prevention of Health Care-Associated Infections. Clin Microbiol Rev. 2016; 29(2):201-22. doi: 10.1128/CMR.00049-15

18. Lowy, F. D. 1998. Staphylococcus aureus infections. N Engl J Med. 1998;339:520-32. 